\title{
Actualización de gestión gerencial eficaz, a partir de la aplicación de nuevas herramientas financieras
}

\author{
Update of effective management management, based on the \\ application of new financial tools
}

\begin{tabular}{|c|c|}
\hline Tibisay Martínez & Aracelis Salavarría \\
\hline $\begin{array}{l}\text { tibisaymartinez@gmail.com } \\
\text { ORCID: 0000-0002-2871-8204 }\end{array}$ & $\begin{array}{l}\text { aracelissalavarria@gmail.com } \\
\text { ORCID: 0000-0002-0163-0202 }\end{array}$ \\
\hline Universidad de Carabobo, Venezuela & Universidad de Carabobo, Venezuela \\
\hline
\end{tabular}

Artículo recibido en mayo 2019 / Arbitrado en junio 2019 / Publicado en septiembre 2019

Palabras clave:

RESUMEN

ABSTRACT

Keywords:
El objetivo general de esta investigación es proponer la aplicación de herramientas de gestión gerencial eficaz, a través de los nuevos argumentos financieros. Como metodología, este estudio se caracteriza de campo con un nivel descriptivo y explicativo. Se aplicó un instrumento a todo el personal que labora en la organización. Una vez aplicado el instrumento, se procesó y analizó. De allí se partió a realizar la matriz DOFA, para diseñar el conjunto de herramientas a aplicar. Se concluyó que es vital para la organización consolidarse de y generar raíces en el mercado donde se desenvuelve, a través de las estrategias que conllevan gerencia eficaz y herramientas financieras, diseñadas para ellos.

Gestión gerencial, gerencia eficaz, herramientas financieras

The general objective of this research is to propose the application of effective managerial management tools, through the new financial arguments. As a methodology, this study is characterized in the field with a descriptive and explanatory level. An instrument was applied to all personnel working in the organization. Once the instrument was applied, it was processed and analyzed. From there, the SWOT matrix was created, to design the set of tools to be applied. It was concluded that it is vital for the organization to consolidate and generate roots in the market where it operates, through strategies that involve effective management and financial tools, designed for them.

Financial Education, Case Method, teaching strategies 


\section{INTRODUCCIÓN}

No se puede dejar a un lado que en la actualidad las organizaciones empresariales se enfrentan a un escenario económico caracterizado por un mercado cambiante, tecnológicamente avanzado y globalizado, por tanto independientemente de su tamaño, se han visto en la necesidad de utilizar cada vez con mayor frecuencia los denominados indicadores de gestión financiera, que están representados por relaciones entre elementos de los estados financieros de la compañía que le permiten obtener información precisa sobre su actualidad, además de las estrategias que implementan para mantenerse competitivo en el ramo donde se desenvuelve.

Así mismo, en la actualidad el proceso de transformación que trae consigo los avances tecnológicos, la automatización de los procesos, el desarrollo económico, el crecimiento de muchas empresas, las variaciones económicas del mercado en el país, dificulta la permanencia y el progreso de cada empresa. Es por eso, que las exigencias implícitas en estos cambios hacen indispensable que las unidades empresariales estén preparadas para gestionar todas las herramientas que le sean necesarias para una verdadera gestión gerencial eficaz.

Sin embargo, no se puede dejar a un lado que las organizaciones son vulnerables a sufrir algún desequilibrio financiero imprevisto, caracterizado por insolvencia y poca liquidez 0 , pero también puede ser producto de políticas financieras poco efectivas 0 por deficiencias en el desempeño estratégico, administrativo, productivo o financiero; por lo tanto, toda empresa debe conocer su condición económica y financiera para identificar los problemas existentes, aunado a plantear estrategias que contribuyan al éxito empresarial, analizando si los factores tienen que ver con el desarrollo de la jornada laboral del personal gerencial con que cuenta.

Belandría (2016: 137) destaca que "..toda empresa requiere de un gerente efectivo, el cual debe seleccionar estrategias y programas que sean plenamente compatibles con el análisis de la situación o realidades que enfrentan la empresa que dirige", esto para el logro de cada uno de los objetivos trazados para esta organización. Por lo cual, se hace trascendental como una manera que permita alcanzarlas metas organizacionales, el análisis e interpretación de cada una de las teorías que conforman los diferentes principios de la administración, en donde se pueda obtener su valor agregado de tal manera comprender realmente el significado de la función gerencial efectiva.

Acorde a esta afirmación, Ortega Castro (2008:2) señala que "una buena Administración Financiera coadyuva a que la compañía alcance sus metas, y a que compita con mayor éxito en el mercado, de tal forma que supere a posibles competidores". Muy importante entonces resulta que la Administración Financiera pueda realizar un diagnóstico preciso de la situación de la empresa, basándose en elementos reales, como lo son sus resultados inmediatamente anteriores para describir qué está sucediendo en su seno y, además de esto, brindar sugerencias orientadas a mejorar cuanto se requiera. Al mencionar las bases utilizadas, se hace referencia a indicadores de gestión 
financiera, los cuáles haciendo uso de relaciones entre resultados de los estados financieros, ofrecen información verídica y confiable de lo que ocurre dentro de la organización; estos indicadores son factores realmente influyentes en relación al éxito que pueda alcanzar en el mercado.

Dentro de este orden de ideas, sacar a relucir efectividad conlleva hacer mención directa de diferentes resultados y cumplimientos, y por ende, es el dar respuesta a la interrogante siguiente: ¿qué tan eficaces y eficientes son los gerentes en las organizaciones?, dentro de las múltiples definiciones, se puede señalar que una persona eficaz, tal como precisa Vázquez (2012:19) "es quien cumple con los objetivos que le fueron encomendados, y usando su creatividad, inteligencia y métodos comúnmente tiene resultados positivos y lo hace de manera reiterada". Cuando una persona, logra objetivos, optimizando los recursos que tuvo a disposición, es cuando se está en presencia de una persona que realmente es eficiente. Por eso, la eficiencia es esencialmente la cualidad de un ser humano de lograr objetivos con economía de recursos.

Es por eso que, la fusión de estas dos cualidades, es decir, eficacia y eficiencia, genera la efectividad, la cual es una cualidad valiosísima para el éxito de las organizaciones. Siendo esto un elemento que todo gerente actual y vanguardista, debe tomar en cuenta, como una manera de alcanzar el ser una persona efectiva en el cargo que ostenta, además de procurar el cosechar la habilidad de tener una conducta asertiva, es decir, ejerciendo el cargo de forma directa, con actitudes honestas y con un trato respetuoso al estar en contacto con las personas que dirige dentro de la empresa.

Es oportuno destacar, que cuando un gerente posee estilo asertivo, resuelve los problemas que se pueden presentar en la empresa, señala Serna (2006) que además está a gusto con todos los trabajadores, permite sentirse satisfecho, por cuanto él lo está consigo; su comportamiento es relajado, con control, crea y fabrica la mayoría de las oportunidades. Asimismo, el lenguaje no verbal de una persona asertiva se caracteriza por sonreír constantemente, manos sueltas y en movimiento, postura erguida, contacto ocular directo y gestos firmes, estas son sus características más resaltantes.

Otro aspecto que se debe considerar, menciona Pantoja (2017) es la creatividad, la cual debe comprenderse como la habilidad de crear algo nuevo $o$ de relacionar algo conocido de una manera innovadora, así también como el alejarse, en muchos casos de esquemas y conductas tradicionales.

Saber establecer y comunicar la estrategia corporativa para alinear los recursos y las personas en una dirección determinada no es tarea sencilla, y un Cuadro de Mando resulta de gran ayuda para lograrlo. A través de sus indicadores de control, financieros y no financieros, se obtiene información periódica para un mejor seguimiento en el cumplimiento de los objetivos establecidos previamente, y una visión clara del desarrollo de la estrategia. Así, y gracias a esta empresarial, la toma de decisiones resulta más sencilla y certera, y se pueden corregir las desviaciones a tiempo. 
Para Rohm del Balanced ScoreCard Institute de EE.UU., el cuadro de mando integral, conocido por sus siglas $\mathrm{CMI}$, debe ser considerado como parte esencial en la gerencia asertiva, al respecto puntualiza Belandría (2016:140), es

...un sistema de administración de desempeño que puede utilizarse en cualquier organización, para alinear la visión y misión con los requerimientos del cliente, las tareas diarias, administrar las estrategias del negocio, monitorear las mejoras en la eficiencia de las operaciones, crear capacidad organizacional, comunicando los progresos a todo el personal.

Ante todo lo planteado, las herramientas gerenciales que son vitales para los verdaderos gerentes en este milenio, son aquellos métodos empleados para hacer más óptima y eficaz la labor de gerencia de una organización, a través de diversos lineamientos que permiten salir adelante $y$ estructurarse en los primeros lugares de la competencia. Dichas herramientas actualmente se encuentran monótonas y un poco repetitivas, sobre todo, a los objetivos que se haya trazado la empresa Saroca, C.A., al inicio del proceso.

Una de las cualidades más útiles para la empresa objeto de estudio, conlleva a la actualización de las herramientas financieras las cuales son muy útiles, porque permiten organizar los estados financieros para llevar un mejor manejo y así tener la seguridad de que se están tomando las mejores decisiones.
La empresa Saroca, C.A. ubicada en Valencia, Venezuela, fue constituida según la forma jurídica como Compañía Anónima (C.A.), hace diez (10) años en enero del año 2008, como iniciativa del Lcdo. Luis Sandoval (Contador-Administrador), el Lcdo. Ricardo Rodríguez (Contador) y el Lcdo. Ernesto Calderón (Msc. Administración Tributaria). Cuenta con una plantilla gerencial conformada por su Presidente, accionistas y su Junta directiva. Adicional a esto cuenta con cuatro Departamentos: Administración y Contabilidad, Compras, Ventas y Operaciones, estos Departamentos están integrados por todas las personas que brindan sus servicios. Saroca, C.A., nace ante la necesidad de empresas, sociedades y compañías de suministro de personal altamente calificado. Forma parte del sector terciario debido a que su principal elemento es la capacidad humana, brindando asesorías en las áreas contables, fiscales, relaciones industriales y jurídicas

Según lo señalado por el Gerente Lcdo. Ernesto Calderón en entrevista sostenida, la organización ha estado dentro del mercado durante diez años, de los cuales los últimos tres años ha presentado fallas de una efectiva comunicación, además de debilidades en el trato entre los subordinados y la gerencia, así como aspectos inherentes a la capacitación que tienen los trabajadores de las diferentes gerencias de la empresa.

Asimismo, no se brinda información detallada y minuciosa de las labores que deben realizarse $y$ no se verifica si se cumplieron según lo señalado a estas asignaciones. Otro aspecto que cabe resaltar, es que de manera constante se evidencian errores en los procedimientos 
administrativos y contables, tales como: mal registro de las operaciones, errores de cálculos numéricos, falta de registros sin los soportes suficientes, fallas en el seguimiento, malas imputaciones presupuestarias, retraso en el registro de documentos administrativos, mal uso del recurso financiero $y$ presupuestario.

Actualmente, su gerencia no cuenta con herramientas actualizadas que le permitan sistematizar el conocimiento de la situación financiera y operacional de la empresa, ya que cuando se necesita información puntual se remiten a resultados de ejercicios anteriores a sus estados financieros para obtenerla, sin la debida implementación del conocimiento de análisis administrativo y financiero, que les permita mantener en uso y disposición de todos los recursos con los cuales cuenta la empresa. Dicho esto, la organización toma datos aislados unos de otros para basar sus decisiones y no realiza un cruce entre la información contenida en cada una de las fuentes, lo cual solo le ha permitido optar por medidas económicas que les han dado un apoyo correcto a corto plazo, concibiendo así que la gerencia no aproveche al máximo su potencial para que las decisiones emanadas del análisis de la información financiera para el beneficio a un largo plazo o si es posible de manera permanente.

Ante todo lo planteado, Saroca, C.A. requiere de la implementación de herramientas financieras que le permitan mejorar la gestión de este particular aspecto, con el objetivo de fomentar la sustentabilidad de su desarrollo y no poner en riesgo su estabilidad.

A tal efecto, la presente investigación tiene como finalidad proponer la aplicación de herramientas de gestión gerencial a través de estrategias de coordinación administrativas, que permitan actualizarse y así optimizar en el proceso de toma de decisiones gerenciales en la empresa Saroca, C.A., ubicada en el municipio Valencia del estado Carabobo, las cuales representarán para la empresa, una base sólida.

Para tal fin se considera necesario diagnosticar los procesos de gestión gerencial de la Empresa Saroca, C.A.; determinar las debilidades, oportunidades, fortalezas y amenazas de la gestión gerencial de la Empresa Saroca, C.A.; y finalmente actualizar las herramientas financieras necesarias para una gestión gerencial eficaz, a través de estrategias de coordinación administrativa, de la empresa Saroca, C.A.

En este sentido, según los criterios de operación y eficacia, los responsables de cada organización son los facultados para decidir qué herramientas son más adecuadas para su modelo de empresa. No es lo mismo, por ejemplo, las estrategias gerenciales de una multinacional que las de un supermercado o de una bodega. En este punto también entra la destreza del gerente para elegir un modelo y acoplarlo a su estructura, aspectos estos que se profundizará a lo largo de este proceso investigativo de manera de proponer a la empresa Saroca, C.A., para que adecúe a los aspectos que sean más beneficiosos para el éxito de la organización.

De esta manera, este estudio promoverá la participación de todos los gerentes de la organización de manera de establecer una verdadera gestión de gerencia eficaz, para mantenerse estables en el mercado, pese a 
la situación económica que está padeciendo la economía de nuestro país en la

\section{MATERIALES Y MÉTODO}

Este estudio se enmarca en una investigación de campo del tipo descriptivo, la cual ha sido definida por Franco (2014: $110)$ como el "método a aplicar para deducir un bien o circunstancia que se esté presentando; se aplica describiendo todas sus dimensiones, en este caso se describe el órgano u objeto a estudiar". Por lo cual, se centrarán en recolectar datos que describan la situación tal y como es.

Según los objetivos propuestos en la presente investigación, la misma será un estudio tipo cuantitativo de corte transversal. En cuanto al indicador cuantitativo, refieren Hernández, Fernández y Baptista (2011), coincidiendo con Pallela y Martíns (2012) que refieren:

...es la expresión matemática que busca reflejar en forma resumida, oportuna, sensible y específica las características de un factor de riesgo, de un factor de protección o de un problema específico en una población dada, en lugar y tiempo determinado. (p. 346 y 96)

Esto conlleva, que son parámetros objetivables y cuantificables que se elaboran a partir de una construcción teórica del aspecto que se intenta medir, con el fin de aplicarlo a toda una población.

Será complementado con una revisión documental con la finalidad de ampliar y profundizar el conocimiento de su naturaleza, con apoyo principalmente, en trabajos previos, información y datos divulgados por medios impresos, audiovisuales o electrónicos.

Por lo tanto, se indaga sobre las herramientas necesarias para una gestión gerencial eficaz, a través de los nuevos mecanismos financieros que requiere la empresa Saroca, C.A. y como en toda investigación, el propósito de este diseño es el aporte de nuevos

La muestra que se utiliza es el total de la población, por ser una cantidad de participantes manejable. Se aplica como instrumento la encuesta y la entrevista. La encuesta se aplica al personal administrativo, los gerentes y accionistas que laboran en la empresa Saroca, C.A. En cuanto a la entrevista, esta consta de un formulario previamente preparado $y$ estrictamente normalizado a través de un conjunto de preguntas establecidas con anterioridad; de esta forma se trazan los límites que la regularán, con el objetivo de conocer la percepción de los entrevistados sobre la situación actual de la empresa Saroca, C.A.

\section{RESULTADOS Y DISCUSION}

La empresa Saroca, C.A. presenta una situación particular en cuanto a su estructura organizacional, lo cual se evidencia en entrevista realizada a su Gerente. Cabe destacar que la empresa se ha visto favorecida por el aumento de la demanda de servicios, ya que la organización está en un proceso de "Planeación estratégica" la cual es una proyección a futuro de lo que queremos hacer y a donde nos proponemos llegar en un largo plazo aprovechando al máximo el potencial que existe, para esto debemos establecer reglas, lineamientos y 
procesos para lograr dicho objetivo en un plazo determinado $y$ en condiciones óptimas, de esta forma la planeación estratégica establece una trayectoria inteligente, buscando áreas de oportunidad, previniendo y evadiendo amenazas, las cuales será acertadamente presentadas en el cumplimiento del objetivo número tres, trazado en este estudio, el cual se presenta en las conclusiones de todo el proceso investigativo.

Esto se debe, en gran parte al mejoramiento en cuanto a las estrategias implementadas, en ellas se definen las acciones que contribuyen a su crecimiento, a su posicionamiento en cada uno de los segmentos del mercado y a alcanzar la rentabilidad esperada. Atender bien a nuestros clientes y brindarles un cordial y amable trato permite el impulso y la calidad del servicio y así se puede generar mejor ingreso económico a nuestra empresa además de una series de estrategias implementadas durante los últimos años, en cuanto a una excelente campaña publicitaria se refiere, dentro del mercado de la ciudad y sus alrededores, lo que le ha permitido estabilidad económica.

En cuanto al aspecto operacional, la empresa ha estado en el mercado desde el año 2008, y durante los tres últimos años, ha experimentado un crecimiento importante. Esto debido al creciente interés por parte de los consumidores de utilizar este servicio, hace que la Gerencia actualmente considere implementar una planeación estratégica, mediante la organización, dirección y control que les permitan afianzar su desarrollo y hacer que el mismo sea constante.

Otro aspecto que resalta, que sus decisiones gerenciales son apoyadas por algunas herramientas financieras, como los estados financieros, presupuestos semestrales. La gerencia sostiene reuniones con una periodicidad semestral, en las que evalúa el desarrollo de sus operaciones y controla el cumplimiento de sus objetivos, manifiestan además, encontrarse en la búsqueda de actualización de herramientas financieras que le permitan estandarizar sus procesos con el objetivo de tener un mayor control de sus actividades y optimizar sus resultado.

Así mismo señalo que la empresa Saroca, C.A. para que continúe funcionando correctamente es necesario solicitar un crédito bancario, de esta manera seguirá cumpliendo con las obligaciones del personal.

El primer objetivo específico precisó el diagnosticar los procesos de gestión gerencial de la empresa Saroca, C.A., siendo las debilidades con mayor ponderación por parte del personal que labora en la empresa objeto de estudio, se tiene:

- Que la inflación de país, repercute en la actitud del trabajador al momento de ejercer su función dentro de la organización, generando estrés al momento de cumplir con las obligaciones asignadas.

- Ausencia de estrategias que motiven acertadamente al personal, de manera que cumplan de sus organizaciones de manera espontánea.

- El personal le cuesta aplicar las instrucciones pertinentes de manera de acatar las órdenes que reciben de sus superiores que conllevan cambios.

- Bajos recursos para optimizar la gerencia. 
- Sostienen los trabajadores de la empresa que no se cuenta con el equipamiento de trabajo que sea pertinente, y que no cuenta con una motivación en cuanto a crecimiento personal se refiere.

Todos estos aspectos destacados son una demostración de que el instrumento arrojó la necesidad de evaluar la labor de la gerencia de la empresa y la necesidad de actualizar los sistemas de información utilizados como herramienta financiera al igual que impartir charlas o brindar material de apoyo para modificar la actitud gerencial y mejorar en esas debilidades de manera de posicionarse como organización exitosa.

Por otro lado, determinar las debilidades, oportunidades, fortalezas y amenazas de la gestión gerencial de la Empresa Saroca, C.A. conlleva a la realización de una matriz DOFA

Cuadro № 1. Matriz DOFA.

\section{Fortalezas (F) Debilidades (D)}

- Buen ambiente laboral.

- Buen trato con los clientes.

- Experiencias de los recursos humanos.

- Conocimiento del mercado.

- Bajos recursos

- Equipamiento de trabajo obsoleto.

- Falta actualizar herramientas financieras.

- Capital de trabajo mal utilizado.

Oportunidades (O)

- Mercado mal atendido.

- Tendencias favorables en el mercado.

- Capacitación técnica del personal.

\section{Estrategias FO}

- Aprovechar el ambiente laboral para el crecimiento del mercado.

- Desarrollar planes para mantenerse en el mercado con los recursos financieros adquiridos.

- Creación de planes con las experiencias laborales aprovechando las tendencias favorables en el mercado.

- Diseños de programas para la capacitación técnica del personal.

\section{Estrategias DO}

- Renovar los equipos de trabajos para obtener una buena organización del mercado - Aprovechar el capital trabajo para la competencia del mercado.

\section{Amenazas (A)}

- Inflación de país.

- No evalúan

- Constantemente los recursos de la empresa.

\section{Estrategias FA}

- Propiciar talleres o cursos para innovar al trabajador.

- Generar nuevos proyectos en pro de la organización.

- Calidad de experiencia de los recursos humanos genera diferencia con la competencia del mercado.

\section{Estrategia DA}

- Proponer herramientas financieras para cumplir las metas.

- Diseños de estructura en el capital de trabajo que permita competir en el mercado.

Fuente: Martínez y Salavarria (2018). 
Este cuadro es una herramienta, la cual contribuye a realizar de manera sistemática el análisis de todos los componentes de la empresa, las cuales están representadas por las condiciones internas y externas de ésta, permitiendo identificar las capacidades positivas y tatar de resolver las negativas que ayudarían a lograr el objetivo y las deficiencias que dificultan su logro.

La empresa Saroca, C.A., como organismo de la administración, permite la prestación de un servicio para satisfacer las necesidades tanto individuales, empresariales, grupales como sociales. La importancia de la organización radica en que permite la conjunción ordenada de recursos humanos, materiales y económicos para el cumplimiento de sus fines. En torno a esto, es necesario que el pensamiento administrativo surja como consecuencia de las necesidades sociales e individuales que se viene gestando por la necesidad de un cambio de paradigma. Los escenarios económicos adquieren un dinamismo muy acelerado y cambiante donde la tecnología, la innovación y la competitividad han dado origen a nuevos retos y exigencia en las ciencias gerenciales con la finalidad de garantizar la supervivencia y el desarrollo empresarial.

Actualmente, para la gerencia, el estado ocupa un papel protagónico determinante para su supervivencia, operatividad, aunado además a todos aquellos cambios que se derivan de la tecnología, la presentación de nuevos servicio, el avance de la ciencia administrativa y sus nuevas herramientas, modelos que se han derivado de la competitividad, obligando a el gerente a lograr un buen desempeño, donde se capacita, y obtenga una visión distinta a lo tradicional, es por ello que el desarrollo del liderazgo es un mundo cambiante, donde se tienen que identificar y desarrollar a personas capaces de dirigir a empresa del siglo XXI.

Las personas son los únicos elementos capaces de dirigirse y desarrollarse por sí mismas, por eso tiene una enorme aptitud para el crecimiento, de allí la necesidad de capacitación para su educación y formación profesional, esto en concordancia a su iniciativa para dirigir y liderar los cambios que surge en la economía moderna.

Según Chiavenato (2007) al referirse al Liderazgo comenta "es fundamental identificar y desarrollar a personas excepcionales, capaces de llevar a la organización hacia el nuevo siglo. Es vital crear líderes de líderes, y el secreto del éxito estará cada vez más en las personas" (pág. 406)

En esta transformación, la gerencia debe estar convencida de los beneficios que acarrean los cambios en los entornos organizacionales, la visión debe estar clara. Ohmae (2005) considera que la función del líder es fundamental para influir en los demás, de estimularlos, de comunicarles la visión y llevarlos al futuro y además es importante mencionar que el líder no tiene que ser una figura solitaria, puede ser un grupo bien definido e integrado en el ideal capaz de comunicar eficientemente lo que se desea. En definitiva, el liderazgo abre paso hacia un nuevo esquema paradigmático de la gerencia, llevando a cabo por líderes que actúan como individuos innovadores al implantar las ideas diferentes al estándar.

Actualmente el crecimiento que ha presentado la empresa Saroca, CA., aunado al creciente interés por parte de los consumidores de utilizar este servicio, hace 
que la Gerencia considere implementar estrategias utilizando indicadores de gestión que les permitan afianzar su desarrollo y hacer que el mismo sea constante. He aquí donde se establecen metas para que sus trabajador puedan alcanzar los objetivos propuestos en sus jornadas diarias, y así poder desempeñar las funciones que le son asignadas, y así ser considerado un buen estratega.

Según el autor Chino SunTzu un buen estratega es aquel que encuentra la manera eficaz para realizar acciones, a partir de datos relevantes al acontecimiento que se suscite.

Como para complementar, desarrollaremos un proceso de planeación estratégicas, que es una forma disciplinada que permite q los gerentes comprendan el ambiente en que opera su organización y de ahí pasar a ejecutar la acción, el proceso consta de dos fases según los autores Hofer y Scherdel: la planeación, en donde se establecer metas, objetivos y planes y la implementación de la estrategia que es la etapa donde se incluyen los pasos de la administración y control estratégicos.

De acuerdo con Rodríguez (2012), se tiene que la planeación es la esencia de la administración, es alcanzar un objetivo, una meta determinada, es decir, es la piedra angular del proceso administrativo debido a que implica conocer la necesidad de acción, investigándola y analizándola, para posteriormente desarrollar una propuesta de acción basada en la investigación, análisis y finalmente, tomar la mejor decisión. (Meggison, 2009, pág. 156)

Puede decirse que planeación consiste en elegir un curso de acción y la toma de decisiones por anticipado de las actividades a realizar, es decir, es la esencia del cuándo y del cómo, de ahí la importancia de que el proceso de planeación se lleve a cabo acertadamente. Ésta secuencia de pasos se compone principalmente de los siguientes elementos: planeación estratégica, táctica y operativa. La primera se realiza a largo plazo, la siguiente a mediano plazo y la última a corto plazo. Dependiendo de la naturaleza de la organización se deberán utilizar y aprovechar un conjunto de planes alineados para su actuación.

La planeación estratégica es el camino o lineamiento general que se adopta para llegar al objetivo planteado, trazado a la par de la visión y misión empresarial mediante el diagnóstico del ambiente externo e interno de la empresa en cuestión. Los principales aspectos de la planeación estratégica influenciados por los gerentes de forma directa son la visión y misión, metas, estrategias y asignación de recursos.

De acuerdo con (Hellriegel, 2015: 183) la planeación táctica implica tomar decisiones específicas y concretas respecto a qué hacer, quién lo hará y cómo lo hará, en un plazo menor o igual a un año, en otras palabras, son los planes de acción con metas establecidas.

Para que exista una empresa, no basta con que haya unos elementos humanos 0 económicos, sino que es preciso disponer de una organización adecuada para la consecución de los objetivos que persigue la empresa dentro de un entorno determinado. En este sentido la organización es la manera de estructurar los elementos humanos y económicos de la empresa para alcanzar los objetivos propuestos en un entorno determinado. 
Para completar la tarea, es preciso conectar correctamente los puestos entre sí,hasta conseguir que el resultado sea similar al que se hubiera obtenido si todo el trabajo pudiera haber sido desarrollado por un único individuo completamente eficiente, quien está realmente preparado para desempeñar tal función. En tal sentido, se propone estrategias de coordinación administrativa para integrar todas las actividades $y$ departamentos de la empresa Saroca, C.A., para así facilitar el trabajo y lograr las metas.

Henry Fayol (1841-1925), de origen francés, el autor más distinguido de la teoría administrativa. Señalo que la teoría administrativa se puede aplicar a toda organización humana (universalidad).

Los Gerentes planean para fijar un rumbo general con la mira puesta en el futuro de la organización (mayores utilidades, participación de mercado más amplia y responsabilidad social). Mediante una organización eficaz los gerentes coordinan mejor los recursos humanos, materiales y de información, una vez que la empresa define a dónde quiere ir y como va hacerlo, el líder debe influir y motivar a los empleados y haciéndole participe en la misión y objetivos. Por último, se llega a las comparaciones, tomar acciones para corregir y retroalimentar.

Se observó que la gran mayoría de los gerentes afirmaron que deben establecer las metas que pretende lograr, en su jornada de trabajo; para obtener una verdadera planificación en la empresa que les permita dictaminar líneas que los subordinados deben seguir. Así mismo, los gerentes coinciden que siempre y algunas veces es que efectúan un análisis de las debilidades encontradas en los procesos administrativos de su departamento , igualmente en cuanto a la evaluación de las estrategias que se debieron cumplir. Denotándose obsolescencia en los sistemas de información que se están aplicando vitales para el adecuado funcionamiento de las organizaciones; las cuales fueron detalladas en el planteamiento del problema.

Es un estudio analítico con diseño documental basado en los fundamentos teóricos de Gitman (2003), Van Horne (2003), Elizondo y Altman (2003), entre otros. El análisis financiero se basa en el cálculo de indicadores financieros que expresan la liquidez, solvencia, eficiencia operativa, endeudamiento, rendimiento y rentabilidad de una empresa.

Se considera que una empresa con liquidez es solvente pero no siempre una empresa solvente posee liquidez. El análisis financiero basado en cifras ajustadas por inflación proporciona información financiera válida, actual, veraz y precisa. Se concluye que el análisis financiero es una herramienta gerencial y analítica clave en toda actividad empresarial que determina las condiciones financieras en el presente la gestión de os recursos financieros disponibles y contribuye a predecir el futuro de la empresa.

En cuanto a la formulación de estrategias alternativas, hay ausencia de planes alternativos como una herramienta de control a ser aplicada en la ejecución estratégica de la planificación financiera, con la finalidad de replantear las acciones para corregir la presencia de alguna deficiencias en el logro de los objetivos o cuando ocurren cambios importantes en los factores competitivos $\mathrm{o}$ en otros elementos del entorno, por lo que se podría inferir, que estas empresas se dirigen en forma reactiva ante las circunstancias y eventos coyunturales presentados. 
Adicionalmente, no manejan el análisis de las desviaciones entre los resultados esperados y los resultados realmente obtenidos, con el fin de analizar sus causas e introducir las correcciones pertinentes, para asegurar el cumplimiento eficiente de los objetivos, metas, de la empresa sobre una base mensual.

Sin embargo el uso de esta herramienta le proporcionaría la información financiera requerida para comparar el desempeño real con el desempeño planificado mostrando cada diferencia como variación favorable o desfavorable del desempeño y así tomarlas medidas correctivas necesarias.

En cuanto al análisis financiero, no se calculan indicadores financieros que expresan la liquidez, solvencia, eficiencia operativa, endeudamiento, rendimiento y rentabilidad, entre otros, siendo esta una herramienta gerencial y analítica clave en toda actividad empresarial que determina las condiciones financieras en el presente, la gestión de los recursos financieros disponibles y contribuye a predecir el futuro de la empresa. Esta herramienta facilita el proceso de toma de decisiones de inversión, financiamiento, planes de acción, permite identificar los puntos fuertes y débiles de la organización, ya que aporta la información necesaria para conocer el comportamiento operativo de la empresa y su situación económica-financiera, para lo cual se fundamenta en los datos expuestos en los estados financieros, que son utilizados para calcular y examinar los indicadores financieros.

Por otra parte, en cuanto a los indicadores de gestión, tampoco incorporan mediciones como complemento para valorar los procesos y resultados de los planes, de las áreas funcionales de las empresas: producción, mercadeo, recursos humanos y finanzas. Estos indicadores cualitativos, son grandes aliados en para la mejora continua y desarrollo de ventajas competitivas, facilitando la toma de decisiones operacionales y estratégicas.

Con respecto a la proceso de toma de decisiones sobre la ejecución estratégica de la planificación financiera, no se maneja de manera oportuna considerando información obtenida del proceso de control. A pesar de la importancia de tomar decisiones en el campo financiero, estas organizaciones difícilmente llevan a cabo un análisis formal sobre la pertinencia de las decisiones financieras implementadas.

Para finalizar, de acuerdo a los resultados de este diagnóstico se puede decir, que el proceso de planificación financiera se realiza de manera deficiente debido a que no se planifican los resultados financieros esperados, existe ausencia de preparación de planes y objetivos estratégicos a corto, mediano y largo plazo, como instrumentos para establecer los escenarios financieros en la organización.

En tal sentido, se puede catalogar como una gestión reactiva, que actúa de acuerdo a los acontecimientos ocurridos sin anticiparse a ellos, pues no consideran los obstáculos en su entorno. Sin embargo, hay que destacar que el diagnóstico evidencia que hay importantes oportunidades para todos los involucrados de mejorar la eficiencia, incrementar la integridad y la transparencia, y aportar valor a la gestión financiera de las PYME para garantizar su sostenibilidad y crecimiento.

\section{CONCLUSIONES}

Un argumento esencial de la competitividad y la sostenibilidad de las 
empresas es su capacidad de innovación que le permita transformar las oportunidades generadas por los desarrollos científicos y tecnológicos en nuevos productos $y$ servicios, y nuevas maneras para competir y lograr ventajas comparativas.

Acorde a la encuesta y entrevista realizada, se pudo determinar que en efecto, la gerencia no maneja indicadores de gestión financiera precisos que muestren la situación actual de la empresa, utilizando resultados de ejercicios anteriores para argumentar sus funciones realizadas. $Y$ de esta forma, ofrecer información realmente útil para la toma de decisiones y poder proyectar sus operaciones.

Los resultados obtenidos indican que la empresa Saroca, C.A. utiliza como estrategias establecer metas en sus jornadas de trabajo, esto le permite alcanzar una excelente planificación administrativa, para así lograr decisiones gerenciales eficaces dentro de la organización.

Con respecto a la utilización de los estados financieros como indicadores de gestión dentro de la organización se afirma que aportan gran utilidad ya que son tomados en cuenta y permite comparar con ejercicios anteriores para realizar la toma de decisiones.

Adicionalmente, se utilizan como factores externos un estudio de mercado para ser considerado dentro del proceso financiero para la toma de decisiones estratégicas y como un mecanismo importante de la gestión financiera.

Por otra parte los resultados indican que en la organización si se toma en cuenta la actualización de los costos operativos y la razón beneficio costo, mientras que los indicadores de gestión de herramientas modernas de la planificación financiera empresarial (calidad total, Reingeniería, Benchmarking, empowerment, cuadro de mando integral y coaching) para la optimización de la gerencia pocas veces son utilizadas.

De esta investigación también se pudo conocer que los gerentes de la empresa Saroca, C.A., no utiliza estrategias propuestas por dicha empresa llevar a cabo sus funciones siendo esto considerado como una debilidad, que aunque tenga estabilidad dentro del mercado la hace ser inferior ante la competencia.

De acuerdo a los resultados obtenidos la empresa Saroca, C.A, no les brinda la oportunidad de superación al personal que allí labora, y eso hace que se sientan desmotivado y que su rendimiento sea bajo, e impida que la gran mayoría de las funciones que realizan no estén acorde a los cargos que desempeñan, al igual que en cuanto a su recompensa salarial se refiere, sienten que no son remunerados como debe ser y eso hace que su ganas de cumplir con sus funciones sea muy opaca.

El desarrollo y crecimiento personal no se da sin un gran esfuerzo, sin valentía para afrontar los cambios y aprender de todas las circunstancias y personas que nos rodean. Por eso es importante tener herramientas de desarrollo y crecimiento personal que podamos utilizar también en el campo personal como en lo profesional.

La empresa Saroca, C.A., se ve en la necesidad de adquirir una nueva obligación financiera, motivado al gran crecimiento que esta ha venido presentando, lo que hace necesario el actualizar y adquirir nuevos activos. Su Presidente señala que esta situación crediticia se encuentra disponible ya que han sido excelentes en cuando al pago de sus obligaciones, por lo tanto esta 
fuente de apalancamiento, se visualiza como una importante herramienta para promover su crecimiento.

\section{REFERENCIAS}

Ackoff, R. (2018). Gerencia Estratégica. Disponible en: https://gustavo-agudelovelez.webnode.es/blog/gerencia/gerenci a-estrategica/conceptos-basicosgerencia/modelos-de-gerenciaestrategica/. Consultado: marzo 11,2018.

Arias, F. (2012). El Proyecto de Investigación: Introducción a la investigación científica. ( $6^{\circ} \mathrm{Ed}$.). Caracas, Venezuela: Episteme C.A

Balestrini, M. (2014). Cómo se elabora el proyecto de investigación: (para los Estudios Formulativos o Exploratorios, Descriptivos, Diagnósticos, Evaluativos, Formulación de Hipótesis Causales, Experimentales y los Proyectos Factibles. Novena edición. Imprenta: Consultores Asociados. Caracas, Venezuela

Baptista, D. (2014). Apoyo en los Sistemas de Gestión Empresariales mediante las Técnicas Multicriterio Dpto. Procesos y Sistemas. Facultad de Ingeniería. Universidad Metropolitana. IX Congreso de Investigación y Creación Intelectual de la Unimet. Caracas, Venezuela. Disponible en: http://ares.unimet.edu.ve/academic/IXco ngreso/documentos/222.pdfConsultado: Diciembre 15, 2017

Belandría, J. (2016). La función gerencial efectiva. Disponible en: https://www.emprendices.co/la-funciongerencial-efectiva-el-gerente-efectivo/. Consultado: Marzo 11, 2018

Caraballo, M. (2015). Indicadores de Gestión Financiera Basados en las Herramientas de la Administración Moderna para las Empresas del Sector Metalmecánico ubicado en Maracay estado Aragua. Universidad De Carabobo. Facultad De Ciencias Económicas y Sociales. Dirección de
Postgrado. Maestría en Administración de Empresas. Mención: Finanzas. Campus La Morita. Disponible en: http://mriuc.bc.uc.edu.ve/bitstream/handl e/123456789/2607/Maestr.pdf.

Consultado: Diciembre 11, 2017

Carrillo, L. (2011). Propuesta para evaluar el sistema de control interno a través de Análisis Financieros Programados de la empresa Técnica Química 86 Nacional, C.A. (Tequinal). Cagua, estado Aragua. Tesis No Publicada. Para optar al título de Especialista en Finanzas

Franco, Y. (2014). Tesis de Investigación. Población y Muestra. Disponible en: http://tesisdeinvestig.blogspot.com/2014/ 06/poblacion-y-muestra-tamayo-ytamayo.html. Consultado: Junio 26, 2017

Hernández, L. (2013). Propuesta de Indicadores de Gestión en la Perspectiva de Innovación y Desarrollo del Balanced ScoreCard (Cuadro de Mando Integral). Caso: Universidad Metropolitana. Maestría en Gerencia Pública. Universidad Metropolitana. Caracas, Venezuela. Disponible: http://ares. unimet.edu.ve/academic/lXcongreso/documentos/119.pdf. Consultado Diciembre 19, 2017

Hernández, R., Fernández, C. y Baptista, P. (2011). Metodología de la investigación. (7ạ Ed.). México, D.F., México: McGraw Hill Interamericana.

Hurtado, L. (2014). La Gerencia Financiera en la Toma De Decisiones. Universidad Militar Nueva Granada. Facultad de Ciencias Económicas. Contaduría Pública. Bogotá, Colombia. Disponible en:

http://repository.unimilitar.edu.co/bitstrea m/10654/12886/1/.pdf. Consultado: Diciembre 11, 2017

Luna, R. (2015). Evaluación De La Gestión Financiera De Empresas Importadoras Mayoristas Del Ramo Ferretero. Período de Estudio Años 2013 - 2014. Universidad de Carabobo. Facultad De Ciencias Económicas y Sociales. Maestría en Administración de 
Empresas. Mención: Finanzas. La Morita. Disponible en: http://mriuc.bc.uc.edu.ve/bitstream/handl e/123456789/2605/Maestr\%C3\%ADa\%2 0Rebeca\%20Luna.pdf?sequence $=1$.

Consultado: Enero 11, 2018.

Madé Serrano, N. (2016). Metodología de la investigación. Editora Mc Graw Hill. México

Nava, M. (2009). Análisis financiero: una herramienta clave para una gestión financiera eficiente. Revista Venezolana de Gerencia (RVG) Año 14. № 48. Universidad del Zulia (LUZ). ISSN 1315. Disponible en: http://www.redalyc.org/ html/290/29012059009/. Consultado: Diciembre 11, 2017

Palella, S. y Martíns, F. (2012). Metodología de la Investigación Cuantitativa.FEDUPEL. Caracas, Venezuela

Pantoja, A. (2017). La comunicación en la gestión gerencial. Disponible en: http://www.monografias.com/trabajos96/c omunicacion-gestiongerencial/comunicacion-gestiongerencial2.shtml\#elclimaora\#ixzz5AsJZS qzh. Consultado diciembre 19,2017

Puerta Vicent, H. (2011). El proceso de investigación. Lo cuantitativo y lo cualitativo. Dirección Electrónica: hernanpuertav@yahoo.com. Disponible en:

http://tecnologiasenlaead.blogspot.com/2 011/04/el-diseno-de-investigacion-ylos.html. Consultado: Junio 26, 2017

Rodríguez, A. (2011). Diseño de Indicadores Financieros para la evaluación de la gestión financiera y la valoración de la empresa Distribuidora de 87 Neumáticos Chirica Vieja, C.A.Tesis No Publicada para optar al título de Magister en Administración de Empresas Mención Finanzas

Rodríguez, E. (2017). Tipos de encuestas. Disponible http://www.tiposde.org/escolares/123tipos-de-encuestas/. Septiembre 5, 2017

Salvatore, F. (2018). Matriz FODA. Somos Venezuela. Disponible en: http://www.matrizfoda.com/dafo/. Consultado: Marzo 11, 2018

Serna, Humberto (2006). Gerencia Estratégica. Octava Edición. 3R Editores. Temas Gerenciales. Universidad de Los Ángeles, Bogotá Colombia.

Tamayo y Tamayo, Mario (2012). El proceso de la investigación científica. ( $5^{\circ} \mathrm{Ed}$.). México, D.F., México: Limusa, S.A

Universidad José Antonio Páez-UJAP (2007). Normas para la Elaboración y Presentación de los Anteproyectos, Proyectos y Trabajos de Grado. San Diego. Carabobo

Universidad Pedagógica Experimental Libertador (2014). Manual de Trabajos de Grado y Especialización. Maestrías y Tesis Corporales. Sexta Edición. Valencia: Ediciones Universidad Pedagógica Experimental Libertador

Vásquez, E. (2012). Efectividad gerencial. Características del gerente efectivo. Disponible en: https://www.gestiopolis.com/ efectividad-gerencial-características-delgerente-efectivo/. Consultado diciembre 19,2017

Vázquez Constantino, E. (2012). La Gerencia Eficaz. Disponible en: https://books.google.co.ve/books?isbn=8 44902384X. Consultado: Enero 11, 2018

Zabala, C. (2012). Evaluación Financiera de la Red de Distribución en las empresas de consumo masivo. Caso KraftFoods de Venezuela, C.A. Tesis No Publicada, para optar al título de Magister en Administración de Empresas Mención Finanzas. Universidad de Carabobo. Disponible en: http://mriuc.bc.uc. edu.ve/bitstream/handle\%C3\%ADa\%.pdf . Consultado: Enero 11, 2018 